\title{
Neurotoxicity of bupivacaine and liposome bupivacaine after sciatic nerve block in healthy and streptozotocin-induced diabetic mice
}

Liljana Markova ${ }^{1,2}$, Nejc Umek², Simon Horvat ${ }^{3}$, Admir Hadžić ${ }^{4,5}$, Max Kuroda ${ }^{4}$, Tatjana Stopar Pintarič́1,2, Vesna Mrak ${ }^{3}$ and Erika Cvetko ${ }^{2^{*}}$

\begin{abstract}
Background: Long-acting local anaesthetics (e.g. bupivacaine hydrochloride) or sustained-release formulations of bupivacaine (e.g. liposomal bupivacaine) may be neurotoxic when applied in the setting of diabetic neuropathy. The aim of the study was to assess neurotoxicity of bupivacaine and liposome bupivacaine in streptozotocin (STZ) induced diabetic mice after sciatic nerve block. We used the reduction in fibre density and decreased myelination assessed by G-ratio (defined as axon diameter divided by large fibre diameter) as indicators of local anaesthetic neurotoxicity.

Results: Diabetic mice had higher plasma levels of glucose $(P<0.001)$ and significant differences in the tail flick and plantar test thermal latencies compared to healthy controls $(P<0.001)$. In both diabetic and nondiabetic mice, sciatic nerve block with $0.25 \%$ bupivacaine $\mathrm{HCl}$ resulted in a significantly greater $\mathrm{G}$-ratio and an axon diameter compared to nerves treated with $1.3 \%$ liposome bupivacaine or saline $(0.9 \%$ sodium chloride) $(P<0.01)$. Moreover, sciatic nerve block with $0.25 \%$ bupivacaine $\mathrm{HCl}$ resulted in lower fibre density and higher large fibre and axon diameters compared to the control (untreated) sciatic nerves in both STZ-induced diabetic $(P<0.05)$ and nondiabetic mice $(P<0.01)$. No evidence of acute or chronic inflammation was observed in any of the treatment groups.

Conclusions: In our exploratory study the sciatic nerve block with bupivacaine $\mathrm{HCl}(7 \mathrm{mg} / \mathrm{kg})$, but not liposome bupivacaine $(35 \mathrm{mg} / \mathrm{kg}$ ) or saline, resulted in histomorphometric indices of neurotoxicity. Histologic findings were similar in diabetic and healthy control mice.
\end{abstract}

Keywords: Bupivacaine hydrochloride, Diabetes, Liposome bupivacaine injectable suspension, Neurotoxicity, Peripheral neuropathy

\footnotetext{
* Correspondence: erika.cvetko@mf.uni-li.si

${ }^{2}$ Institute of Anatomy, Faculty of Medicine, University of Ljubljana, Korytkova ulica 2, 1000 Ljubljana, Slovenia

Full list of author information is available at the end of the article
}

(c) The Author(s). 2020 Open Access This article is licensed under a Creative Commons Attribution 4.0 International License, which permits use, sharing, adaptation, distribution and reproduction in any medium or format, as long as you give appropriate credit to the original author(s) and the source, provide a link to the Creative Commons licence, and indicate if changes were made. The images or other third party material in this article are included in the article's Creative Commons licence, unless indicated otherwise in a credit line to the material. If material is not included in the article's Creative Commons licence and your intended use is not permitted by statutory regulation or exceeds the permitted use, you will need to obtain permission directly from the copyright holder. To view a copy of this licence, visit http://creativecommons.org/licenses/by/4.0/ The Creative Commons Public Domain Dedication waiver (http://creativecommons.org/publicdomain/zero/1.0/) applies to the data made available in this article, unless otherwise stated in a credit line to the data. 


\section{Background}

The incidence of diabetes has been steadily increasing, and in the next two decades it is estimated that over 640 million people worldwide will be affected [1]. Although only $10 \%$ of patients with diabetes report symptomatic peripheral neuropathy, as many as 50\% may already have subclinical neuropathy [2]. Patients with diabetes require surgical procedures more frequently than healthy patients, and due to their comorbidities, peripheral nerve blocks are often recommended as an alternative to general anaesthesia, particularly for lower extremity surgery [3]. In diabetic rats, a prolonged application of high doses of local anaesthetics perineurally has been associated with neurotoxicity [4-6]. In humans, it is not well-established if nerve blocks can exacerbate a pre-existing diabetic neuropathy [7].

Extended-release local anaesthetic formulations have been recently developed to increase the duration of peripheral nerve blocks and to reduce the risk of systemic or local tissue toxicity [8]. Bupivacaine liposome injectable suspension (DepoFoam bupivacaine, EXPAREL ${ }^{\oplus}$, Pacira Pharmaceuticals, Inc., San Diego, CA, USA) is an extended-release formulation of bupivacaine encapsulated in multivesicular liposomes that has been approved by the U.S. Food and Drug Administration for wound infiltration and interscalene brachial plexus block $[9,10]$. Studies to date have found no evidence of neurotoxicity of liposome bupivacaine used for peripheral nerve blocks or epidural applications in animals and humans [11-17]. However, long-acting local anaesthetics (e.g. bupivacaine $\mathrm{HCl}$ ) or sustained-release formulations of bupivacaine (e.g. liposome bupivacaine) could prove neurotoxic in the presence of a pre-existing neuropathy [5]. The aim of this study was to assess the neurotoxic effects of liposome bupivacaine and bupivacaine hydrochloride $(\mathrm{HCl})$ following perineural injection for sciatic nerve block in streptozotocin (STZ)-induced diabetic mice. We hypothesized that perineural injections of bupivacaine $\mathrm{HCl}$ and liposome bupivacaine would result in a reduction of fibre density and decreased myelination in diabetic nerve.

\section{Results}

\section{Animal characterization}

Prior to induction of diabetes, no significant difference in mean body mass was observed between the diabetic [24.6 (1.5) g] and nondiabetic groups [24.7 (2.0) g]. Four weeks after STZ treatment, a lower mean body mass [21.3 (1.9) g] was recorded in diabetic compared to nondiabetic group $[27.7(2.1) \mathrm{g}],(P<0.001)$. At the same time, fasting glucose levels were higher in diabetic [32.4 (2.0) $\mathrm{mmol} \mathrm{l}^{-1}$ ] compared to nondiabetic mice [6.8 (0.9) $\left.\mathrm{mmol} \mathrm{l}^{-1}\right](P<0.001)$.
Before application of STZ, no differences were noted in paw withdrawal test thermal latencies between the groups. By contrast, following the STZ treatment and prior to sciatic nerve block, significant differences were observed in tail flick and plantar test thermal latencies between the groups (Fig. 1). The success of sciatic nerve block was confirmed in all animals using a paw withdrawal test.

\section{Histopathological evaluations}

For each animal, the treated and untreated sciatic nerve tissue specimens were analysed. Data are presented in Table 1. After bupivacaine $\mathrm{HCl}$ treatment, the sciatic nerves of diabetic and nondiabetic mice showed a significantly lower fibre density compared to the control (untreated) sciatic nerves, while lower myelin width, and higher axon and large fibre diameters was observed compared to the saline treated nerves. After liposome bupivacaine and saline treatments, by contrast, no differences were observed in morphometric parameters compared to untreated control nerves in both diabetic and nondiabetic mice. Thus, the presence of diabetes did not affect the severity of morphometric changes among the groups (Fig. 2). There was also no evidence of inflammation observed in any specimen; inflammatory cells were scarce, occurring only as discrete leucocytes in a few specimens (Fig. 3).

\section{Discussion}

In our study in mice, the sciatic nerve block with bupivacaine $\mathrm{HCl}$, but not liposome bupivacaine, reduced the nerve fibre density and increased the G-ratio, suggestive of demyelinating neuropathy. Under the conditions of our study, the presence of STZ-induced diabetic neuropathy did not appear to affect the severity of pathophysiological changes of nerves treated with bupivacaine $\mathrm{HCl}$ or liposome bupivacaine.

Previous studies in healthy rats reported no histologic changes indicative of neurotoxicity after application of bupivacaine $\mathrm{HCl}$ and liposome bupivacaine $[11,13,16]$. Both local anaesthetic formulations were also reported to be safe for use in brachial plexus nerve block in rabbits and dogs [12], and in sciatic nerve block in pigs [14]. Similarly, a summary of clinical trials of off-label liposome bupivacaine use for peripheral nerve block in 335 healthy patients without neuropathy concluded that liposome bupivacaine had a similar safety and side effect profile to bupivacaine $\mathrm{HCl}$ and saline [15].

However, prolonged exposure of neuropathic nerves to long-acting (bupivacaine) or prolonged-release local 

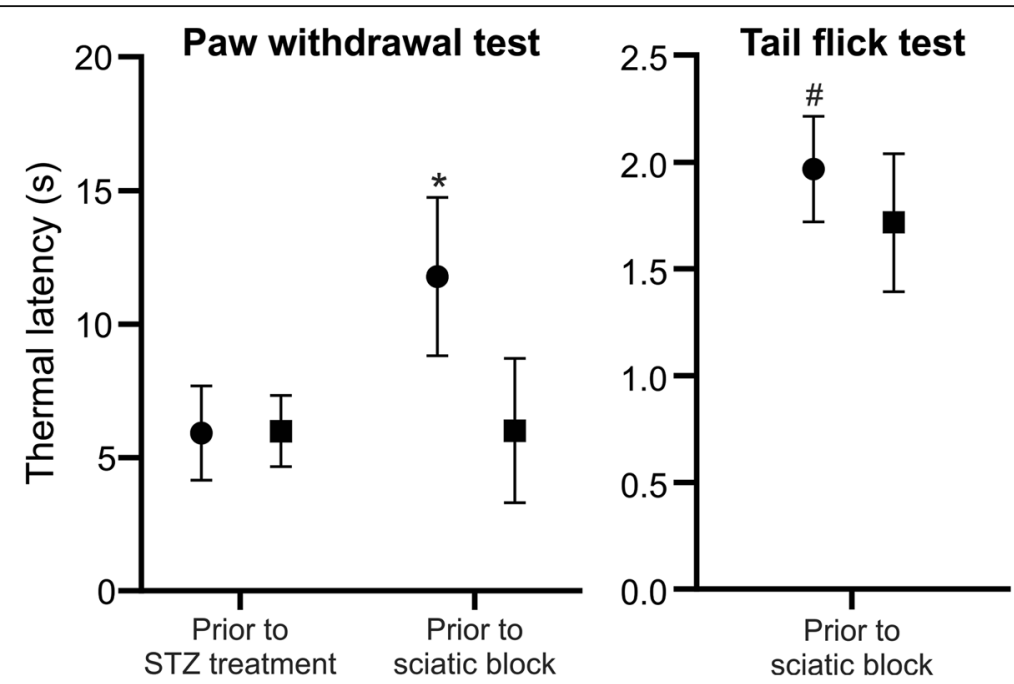

Fig. 1 Paw withdrawal test before streptozotocin (STZ) treatment and two days prior to sciatic nerve block and tail flick test two days prior to sciatic nerve block in STZ-induced diabetic $(\bullet)(n=18)$ and nondiabetic $(\mathbf{\square})(n=18)$ mice. ${ }^{*} P<0.0001$ vs. nondiabetic mice prior to sciatic block and diabetic and nondiabetic mice prior to STZ treatment (one-way ANOVA); $\# P<0.001$ vs. nondiabetic mice (independent $t$-test)

anaesthetic formulations (liposome bupivacaine) may result in neurotoxicity. Peak local anaesthetic concentration may also play a role in nerve injury in subjects with diabetic neuropathy [12]. In STZ-induced diabetic rats, application of $2 \%$ and $4 \%$ but not $1 \%$ lidocaine resulted in nerve oedema, degeneration and demyelination of myelinated nerve fibres. [18]. These data led to the teaching that the risk for local anaesthetic-induced nerve injury could be higher in animals and subjects with diabetic neuropathy [4]. Myelin sheet thinning was documented after application of $0.5 \%$ ropivacaine, $1 \%$ lidocaine with clonidine, and 1\% lidocaine with epinephrine in STZ-induced diabetic rats [5]. Moreover, application of $2 \%$ lidocaine may also be a cause of neurotoxicity in obese diabetic rats even with subclinical diabetic neuropathy [19]. The duration of the local anaesthetics exposure could also contribute to neurotoxicity [5].

The available reports on the neurotoxic effects of local anaesthetic [20-22] suggest that neurotoxicity is not consistent and may depend on the model, mode of application, type of local anaesthetic and other factors. With liposome bupivacaine, however, the delayed release of free bupivacaine from the selected dose of liposome bupivacaine in our study may not have reached the local tissue concentration level capable of causing neurotoxicity [23]. The pharmacokinetic studies indicate that the release of free bupivacaine from liposome bupivacaine is not linear; the bupivacaine releases only after $12 \mathrm{~h}$. However, since the free bupivacaine release occurs over $72 \mathrm{~h}$, its local tissue concentration may be too small to cause neurotoxicity. Indeed, the pharmacokinetic data from clinical studies indicate that the release of the local anaesthetic from the formulation is low, resulting in light sensory, and no motor block [24].

Diabetic neuropathic nerves exhibit complex functional changes [7]. In our study, diabetic mice showed early functional sensory impairment without morphological correlates, consistent with previous findings in STZ-induced diabetic rats $[5,25,26]$. In contrast, in 6week-old male STZ-induced diabetic mice of the same strain used in another study, thin, disorganized and demyelinated sciatic nerve fibres were observed [27]. Given that axon and myelin sheet growth are not yet completed in the 6 week old mice [28], the difference in animal age at the time of STZ application (6 weeks in Pan et al. [27] and 8 weeks in our study), may be responsible for the differential effects of STZ and hyperglycaemia on the sciatic nerves.

In our study, the number of small sciatic nerve fibres was lower following bupivacaine $\mathrm{HCl}$ compared to saline application in nondiabetic mice. However, this effect was not replicated in other morphometric studies after perineural bupivacaine $\mathrm{HCl}$ application in rat, rabbit or dog model [11-13]. Given that the small nerve fibres are usually first affected by diabetes [29], bupivacaine $\mathrm{HCl}$ may cause small nerve fibre degeneration after nerve blockade in the setting of diabetic neuropathy.

We did not find any signs of inflammation in diabetic and nondiabetic nerves, which is consistent with the observations reported by McAlvin et al. [13]. In contrast, using an open approach for sciatic nerve block in nondiabetic rats, infiltrations with macrophages, lymphocytes and fibroblasts have been observed after both liposome bupivacaine and bupivacaine $\mathrm{HCl}$ injections [11]. 
Table 1 Histomorphometric parameters of the sciatic nerve after treatment with bupivacaine hydrochloride (BHCl), liposome bupivacaine (LB) and saline in STZ-induced diabetic mice and nondiabetic control mice

\begin{tabular}{|c|c|c|c|c|c|c|c|}
\hline & & \multicolumn{3}{|l|}{ Treated nerves } & \multicolumn{3}{|l|}{ Control nerves } \\
\hline & & Saline & $\mathrm{BHCl}$ & LB & Saline & $\mathrm{BHCl}$ & LB \\
\hline \multirow[t]{2}{*}{ Fibre density $\left(\mathrm{mm}^{-2}\right)$} & Diabetic & $23724(6888)$ & $22862(4349)^{\dagger}$ & $30259(8511)$ & $26232(4743)$ & 31717 (8258) & $23347(2872)$ \\
\hline & Nondiabetic & $22492(4881)$ & $21582(6144)^{\dagger+}$ & $21983(3408)$ & $25969(2950)$ & $29522(3558)$ & $25906(5004)$ \\
\hline \multirow[t]{2}{*}{ Large fibre area per total area } & Diabetic & $65.20(2.54)$ & $62.63(7.00)$ & $64.03(2.50)$ & $67.93(1.39)$ & $64.58(3.00)$ & $67.64(2.00)$ \\
\hline & Nondiabetic & $66.91(3.61)$ & $63.95(6.46)$ & $65.71(2.53)$ & $65.87(2.39)$ & $65.19(2.86)$ & $67.72(1.90)$ \\
\hline \multirow[t]{2}{*}{ Large fibre diameter ( $\mu \mathrm{m})$} & Diabetic & $5.58(0.65)$ & $\begin{array}{l}5.58 \\
(0.54)^{\dagger}\end{array}$ & $4.97(0.72)$ & $5.37(0.48)$ & $4.81(0.68)$ & $5.62(0.29)$ \\
\hline & Nondiabetic & $5.82(0.69)$ & $\begin{array}{l}5.8 \\
(0.75)^{\dagger+}\end{array}$ & $5.68(0.42)$ & $5.27(0.38)$ & $4.94(0.21)$ & $5.42(0.59)$ \\
\hline \multirow[t]{2}{*}{ Axon diameter ( $\mu \mathrm{m})$} & Diabetic & $3.13(0.41)$ & $\begin{array}{l}3.53 \\
(0.31)^{\#++}\end{array}$ & $2.79(0.68)$ & $2.97(0.55)$ & $2.85(0.31)$ & $3.36(0.32)$ \\
\hline & Nondiabetic & $3.26(0.49)$ & $3.55(0.50)^{\dagger+}$ & $3.25(0.17)$ & $2.98(0.09)$ & $2.78(0.16)$ & $3.04(0.27)$ \\
\hline \multirow[t]{2}{*}{ Myelin width ( $\mu \mathrm{m})$} & Diabetic & $1.23(0.18)$ & $1.01(0.17)^{*}$ & $1.09(0.06)$ & $1.14(0.12)$ & $1.06(0.19)$ & $1.14(0.09)$ \\
\hline & Nondiabetic & $1.28(0.19)$ & $1.12(0.29)^{*}$ & $1.21(0.22)$ & $1.15(0.20)$ & $1.08(0.07)$ & $1.19(0.20)$ \\
\hline \multirow[t]{2}{*}{ G-ratio (axon diameter/large fibre diameter) } & Diabetic & $0.56(0.03)$ & $0.63(0.04)^{* * \# \#}$ & $0.56(0.06)$ & $0.55(0.06)$ & $0.60(0.09)$ & $0.60(0.04)$ \\
\hline & Nondiabetic & $0.56(0.04)$ & $\begin{array}{l}0.62 \\
(0.06)^{* *} \# \#\end{array}$ & $0.58(0.05)$ & $0.57(0.04)$ & $0.56(0.02)$ & $0.56(0.03)$ \\
\hline
\end{tabular}

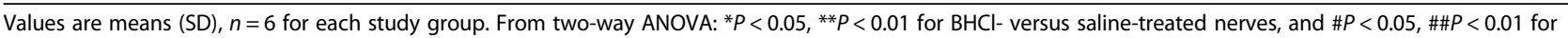
$\mathrm{BHCl}$ - versus $\mathrm{LB}$-treated nerves. From dependent $\mathrm{t}$ test: $\mathrm{t} P<0.05$, $\dagger+P<0.01$ between treated and control nerves

Our results should not be directly extrapolated to the clinical practice of perineural application of bupivacaine and liposome bupivacaine, due to a number of limitations. First, being the first study on nerve blocks in diabetic mice with bupivacaine and liposome bupivacaine, we used a single, exploratory dose and concentration. In addition, the observed changes in our study may be specific to our animal model and nerve block technique. We used a percutaneous block technique to minimize nerve inflammation due to the procedure [5]. Furthermore, inflammatory changes in a STZ-induced diabetic model may be diminished, because STZ depletes immune cells in the peripheral nervous system up to 3 weeks after treatment [30]. A longer-term longitudinal

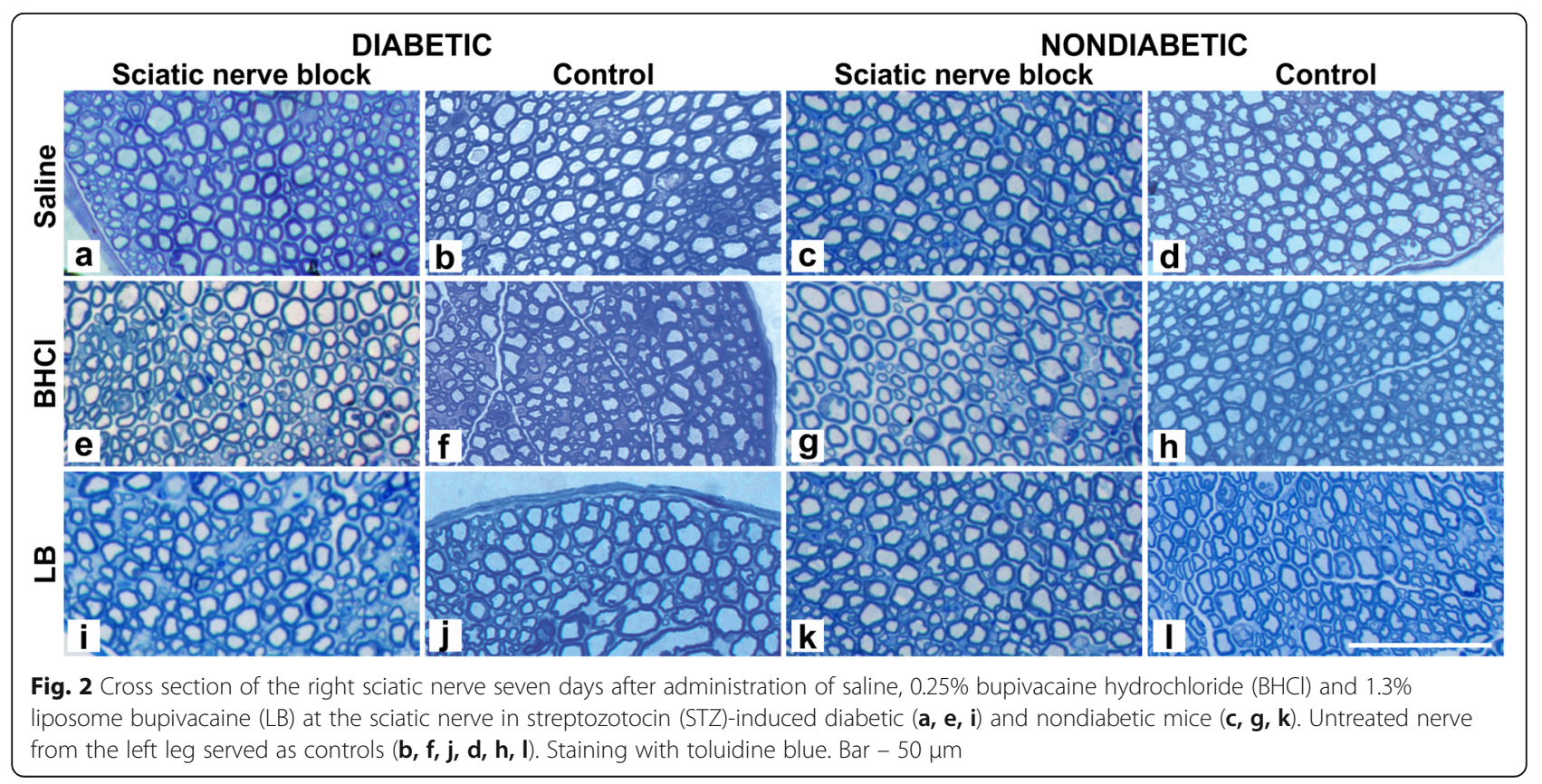




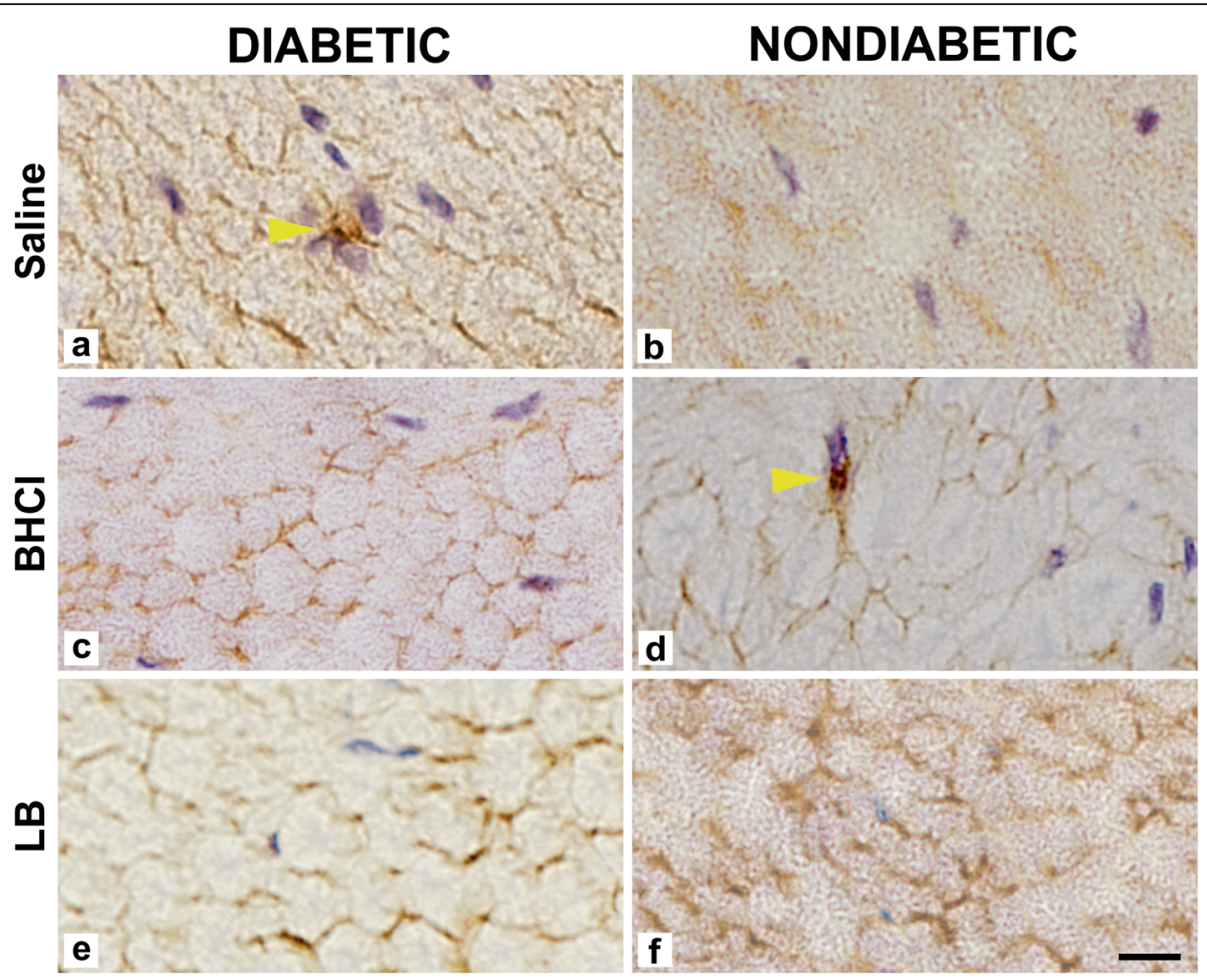

Fig. 3 Cross-section of the right sciatic nerve seven days after administration of saline, 0.25\% bupivacaine hydrochloride (BHCl) and $1.3 \%$ liposome bupivacaine (LB) at the sciatic nerve in STZ-induced diabetic (a, $\mathbf{c}, \mathbf{e})$ and nondiabetic mice (b, d, f) demonstrating rare leucocyte (arrows) infiltration. Immunoreactivity for CD45 is presented. Bar - $50 \mu \mathrm{m}$

study in a high-fat-diet-induced diabetes type- 2 model could be more informative in assessing possible inflammatory effects.

The unifascicular sciatic nerve in mice may be more sensitive to neurotoxic effects compared to multifascicular nerves with abundant connective tissue within epineurium in humans. Although large animal models may better resemble the multifascicular sciatic nerve seen in humans, there are difficulties in establishing diabetes and diabetic neuropathy in larger animals [31]. Further, the STZ-induced diabetic mouse model does not correlate well with all aspects of type- 1 or type- 2 diabetes in humans [30]. High-fat-diet-induced diabetes is thought to better represent the more prevalent type- 2 diabetes in humans; however, there is no diabetic mouse model that intimately mirrors the human pathophysiology of diabetes [32]. And finally, given that only females were used in our study, another study in males is warranted as sex differences in STZ sensitivity have been noted in rodent models [33]. Our study should be viewed as an exploratory or a pilot experiment. However, we believe that our data could be useful to inform future studies and investigators in structuring more robust, dose-ranging studies on neurotoxicity in diabetic mice.

\section{Conclusions}

Under the conditions of our study, the preliminary data suggest that application of bupivacaine $\mathrm{HCl}$, but not liposome bupivacaine, resulted in histological evidence of neurotoxicity in both STZ-induced diabetic and nondiabetic mice.

\section{Methods}

The study was carried out in accordance with the recommendations of the Guide for the Care and the Use of Laboratory Animals of the National Institutes of Health (National Research Council (U.S.) [34], the Committee for the Update of the Guide for the Care and Use of Laboratory Animals, and the Institute for Laboratory Animal Research (U.S.), 2011). The study was approved by the Ethical Committee for laboratory animals of the Republic of Slovenia (Permit Number: U34401-21/2013/6) following European directives on the use of laboratory animals in research and the ARRIVE guidelines.

\section{Animal housing and induction/confirmation of diabetes} Six weeks old C57BL/6J-OlaHsd female mice $(n=36$, weight 25-30 g) were obtained from Harlan Laboratories - Envigo (Italy) and reared at the Centre for Laboratory 
Animals of the Biotechnical Faculty of the University of Ljubljana. All mice were housed individually in ventilated cages (IVC system) with temperature maintained at $23 \pm 1^{\circ} \mathrm{C}$, humidity maintained at $40-60 \%$, and a 12 hour light/12-hour dark cycle.

At the age of 8 weeks, after 2 weeks of quarantine and acclimatization period with free access to clean water and standardized diet (Mucedola, Milan, Italy), diabetes type 1 was induced in mice by intraperitoneal injection of $200 \mathrm{mg} \mathrm{kg}^{-1} \mathrm{STZ}$ in accordance with the protocols for achieving STZ-induced diabetes in mice [27, 35]. STZ is an alkylating agent that induces degeneration in pancreatic $\beta$ islets $[18,36]$. Diabetes was confirmed by measuring a fasting glucose level using Bayer Contour glucose meter (Ascensia Diabetes Care Holdings AG, Switzerland) three weeks after STZ injection. Animals with a fasting glucose level of more than $25 \mathrm{mmol} \mathrm{l}^{-1}$ were considered diabetic, while those with less than $8 \mathrm{mmol} \mathrm{l}^{-1}$ were considered nondiabetic [27]. All STZ induced diabetic mice included in the study met the criteria for diabetes.

\section{Verification of diabetic neuropathy}

To confirm the presence of peripheral sensory neuropathy, tail flick and paw withdrawal tests were performed using Combination Plantar/Tail Flick Analgesia Meter (IITC Life Science, California, USA) with infrared intensity set at $40 \%$ and $50 \%$, and cut-off times of 4.00 and $15.00 \mathrm{~s}$, respectively [37]. The paw withdrawal test was performed two days before the STZ application and two days prior to the sciatic nerve block, whereas the tail flick test was performed two days prior to the sciatic nerve block. Heat stimulation was repeated 3 times at 5 min-intervals; the mean value of the two measurements was used as the baseline [38]. The plantar method is based on Hargreaves method of quantifying the heat thresholds in the hind paws of rodents upon application of radiant or infrared heat stimulus [39]. The tail flick test involved the application of a heat stimulus to the tail after which the time for the tail to "flick" or twitch was recorded. We used a tail temperature option with an automatic temperature trigger at the start of the tests. Once the pre-set temperature was reached, the timer was automatically triggered and stopped after the tail flicks and the light had stopped. The automatic readouts of the start and end temperatures, and the test time improved a repeatability of the measurements. This option has solved the problem associated with "tail temperature prior to and at the end of testing" [40]. While the recent reviews discussed advantages as well as disadvantages of both tests, the two methods are still considered as relevant stimulusevoked nociception tests [41].

\section{Study groups}

Eighteen STZ-induced diabetic and eighteen nondiabetic mice were randomized into the three treatment groups. According to the group assignment, both diabetic and nondiabetic groups included 6 mice treated with $35 \mathrm{mg}$ $\mathrm{kg}^{-1} \quad 1.3 \%$ liposome bupivacaine (EXPAREL), 6 mice treated with $7 \mathrm{mg} \mathrm{kg}^{-1} 0.25 \%$ bupivacaine $\mathrm{HCl}$ (AstraZeneca UK Ltd, UK), and 6 mice treated with saline ( $\mathrm{NaCl}$ Braun, $9 \mathrm{mg} \mathrm{ml}^{-1}$ injection solution, B Braun Melsungen AG, Germany).

\section{Sciatic nerve block}

The mice were anaesthetized with isoflurane up to $4 \%$ in a nitrous oxide/oxygen mixture $(\mathrm{N} 2 \mathrm{O} / \mathrm{O} 2)$ via a facemask. Sciatic nerve blocks were performed by injecting local anaesthetics or saline perineurally using a 29-gauge needle (Omnican ${ }^{\circ}$ A, B. Braun Melsungen AG, Germany) while held in a lateral recumbent position with paws in a right angle with the trunk. The needle was introduced posteromedially towards the greater trochanter in an anteromedial direction. After encountering the ischial tuberosity, $85 \mu \mathrm{l}$ of testing solution was injected by a single trained research staff member, blinded to the study group assignment $[13,42,43]$. The success of the sciatic nerve block was evaluated $20 \mathrm{~min}$ after using the paw withdrawal test.

\section{Histopathological evaluation of the sciatic nerve}

The animals were sacrificed by cervical dislocation one week after the nerve block in order to allow enough time for nerve pathohistological changes to manifest [44]. At the site of local anaesthetic injection and contralaterally, five mm-long sections of the sciatic nerve were harvested and processed for Epon-embedding for histomorphometric evaluations. After initial fixation in Karnovsky's KII Solution (2.5\% glutaraldehyde, $4.0 \%$ paraformaldehyde in $0.1 \mathrm{M}$ sodium cacodylate buffer, $\mathrm{pH} 7.4$ ), the nerve sections were post-fixed in an 1:1 solution of $2 \%$ aqueous osmium tetroxide and $3 \%$ potassium ferrocyanide. Dehydration was accomplished with graded ethanol solutions and propylene oxide following Epon embedding. A high-resolution light microscope (Eclipse E800; Nikon, Tokyo, Japan) was used to study the prepared $0.5 \mu \mathrm{m}$ toluidine blue stained crosssections with images captured by a digital camera (DXM1200F $^{\text {rs }}$, Nikon, Tokyo, Japan) connected to the microscope. Images were analysed by a single operator blinded to group assignment.

Morphometric analysis was performed using the Ellipse program (ViDiTo, version 2.0.7.1, 2004, Košice, Slovakia) [14]. Randomly selected areas of the nerve were analysed. The outer border of the nerve fibres and the inner border of the myelin sheaths were assessed at high magnification followed by measurement of the nerve fibre density, 
proportion of large fibres (percent of fibres where the myelin sheet is visible and can be circumscribed), large fibre diameter, axon diameter and myelin width. Furthermore, G-ratio defined as axon diameter divided by large fibre diameter of the myelin sheath was also calculated [45]. The images were analysed by a trained evaluator blinded to group assignment.

Histopathological evaluation was also employed to assess inflammatory cell infiltration in the histological specimens. Frozen samples of the sciatic nerve were sliced into $10 \mu \mathrm{m}$ transverse sections processed for immunohistochemistry for leucocyte receptor-type tyrosine-protein phosphatase C (CD45) labelling with anti-CD45 antibody (MCA1388, Bio-Rad Laboratories Inc., San Francisco, CA, USA) and revealed by a secondary antibody P0260 (Dako, Glostrup, Denmark). Positive and negative tissue controls were included with each batch of slides as a check on correct tissue preparation and staining techniques. Sections of mouse thymus served as positive control for the presence of leukocytes. For negative controls, the sections in which the primary antibody was replaced with phosphate-buffered saline were used. The images were analysed by a trained evaluator blinded to group assignment.

\section{Statistical analysis}

The Shapiro-Wilk test was used to evaluate the groups for normality. If normality and equal variance assumptions were met, differences in histomorphometric parameters among treatment groups were tested by twoway analysis of variance (ANOVA) followed by Bonferoni post-hoc tests that corrected the $p$-values for the subgroup analyses. The dependent $t$-test for paired samples was used to test differences in histomorphometric parameters between treated and untreated sciatic nerves in the same animal. One-way ANOVA, followed by Tukey post-hoc tests was used for paw withdrawal test. Independent $t$-test was used to compare tail flick test results, body mass and fasting glucose. Statistical analysis was performed with the IBM SPSS Statistics for Windows, version 25 (IBM Corp, NY, USA). Differences were deemed statistically significant at $P<0.05$. Data are presented as means (standard deviation).

The sample size calculation was based on the primary research hypothesis that the STZ-induced diabetic and nondiabetic nerves would differ in their fibre density as an indicator of local anaesthetic neurotoxicity [46]. Using the difference in mean fibre density $(14,000$ fibres per $\mathrm{mm}^{2}$ ), pooled standard deviation (1600 fibres per $\mathrm{mm}^{2}$ ), Type I alpha (0.01), and a desired power (0.90), the sample size was estimated at 6 animals in each treatment group for this two-sided test of a completely crossed $2 \times 3$ ANOVA (diabetic/nondiabetic by liposome bupivacaine/bupivacaine $\mathrm{HCl} /$ saline).

\section{Abbreviations}

ANOVA: Analysis of variance; $\mathrm{BHCL}$ : Bupivacaine hydrochloride; $\mathrm{HCl}$ : Hydrochloride; IVC: Individually ventilated cages; LB: Liposome bupivacaine; STZ: Streptozotocin

\section{Acknowledgements}

We are thankful to I. Blazinovic, M. Crnak Maasarani, M. Slak, N Pollak Kristl, F. Stendler, M. Stevanec, and A. Vidmar from the Institute of Anatomy, Faculty of Medicine, University of Ljubljana, Ljubljana, Slovenia, to Dr. Marija Damjanovska, University Clinical Centre Ljubljana, for technical support; and to Miha Pintaric and Chiedozie K Ugwoke for manuscript proofreading.

\section{Authors' contributions}

LM, SH, EC designed the study, conducted the study, collected data, wrote the manuscript, accepted the final version; VM, TSP collected data, wrote the manuscript, accepted the final version; NU analysed data, wrote the manuscript, accepted the final version; $\mathrm{AH}, \mathrm{MK}$ designed the study, wrote the manuscript, accepted the final version. EC created the laboratory images. All authors have read and approved the manuscript.

\section{Funding}

This work was supported by the Slovenian Research Agency grants (P3-0043, P4-0220) and tertiary funding from the Clinical Department of Anaesthesiology and Intensive Therapy, University Clinical Centre (Ljubljana, Slovenia). The funder had no role in study design, data collection and analysis, decision to publish, or preparation of the manuscript.

\section{Availability of data and materials}

The datasets used and/or analysed during the current study are available from the corresponding author on reasonable request.

\section{Ethics approval and consent to participate}

The study was carried out in accordance with the recommendations of the Guide for the Care and the Use of Laboratory Animals of the National Institutes of Health (National Research Council (U.S.) [34], the Committee for the Update of the Guide for the Care and Use of Laboratory Animals, and the Institute for Laboratory Animal Research (U.S.), 2011). The study was approved by the Ethical Committee for laboratory animals of the Republic of Slovenia (Permit Number: U34401-21/2013/6) following European directives on the use of laboratory animals in research and the ARRIVE guidelines.

\section{Consent for publication}

Not applicable.

\section{Competing interests}

Dr. Hadzic has consulted and/or performed sponsored research for Philipps, Pacira, BBraun Medical, and Heron Therapeutics. He owns and manages NYSORA.com (education), MedXpress.Pro (IP), and VisionExpo,Design (medical design). The other authors declare no competing interests.

\section{Author details}

'Department of Anaesthesiology and Surgical Intensive Therapy, University Medical Centre Ljubljana, Zaloška cesta 7, 1000 Ljubljana, Slovenia. ${ }^{2}$ Institute of Anatomy, Faculty of Medicine, University of Ljubljana, Korytkova ulica 2, 1000 Ljubljana, Slovenia. ${ }^{3}$ Department of Animal Science, Biotechnology and Immunology, Biotechnical Faculty, University of Ljubljana, Groblje 3, 1230 Domžale, Slovenia. ${ }^{4}$ NYSORA, 2581, Broadway, New York, NY 10025, USA. ${ }^{5}$ Ziekenhuis Oost-Limburg, Schiepse Bos 6, Genk 3600, Belgium.

Received: 20 March 2020 Accepted: 6 July 2020

Published online: 17 July 2020

\section{References}

1. Zimmet PZ, Alberti KGMM. Epidemiology of diabetes - Status of a pandemic and issues around metabolic surgery. Diabetes Care. 2016;39:878-83.

2. Gregg EW, Sorlie P, Paulose-Ram R, Gu Q, Eberhardt MS, Wolz M, et al. Prevalence of lower-extremity disease in the U.S. adult population $\geq 40$ years of age with and without diabetes: 1999-2000 National Health and Nutrition Examination Survey. Diabetes Care. 2004;27:1591-7. 
3. Dhatariya K, Levy N, Kilvert A, Watson B, Cousins D, Flanagan D, et al. NHS Diabetes guideline for the perioperative management of the adult patient with diabetes. Diabet Med. 2012;29:420-33.

4. Kalichman MW, Calcutt NA. Local anesthetic-induced conduction block and nerve fiber injury in streptozotocin-diabetic rats. Anesthesiology. 1992;77:941-7.

5. Kroin JS, Buvanendran A, Williams DK, Wagenaar B, Moric M, Tuman KJ, et al. Local anesthetic sciatic nerve block and nerve fiber damage in diabetic rats. Reg Anesth Pain Med. 2010;35:343-50.

6. Lirk P, Verhamme C, Boeckh R, Stevens MF, Ten Hoope W, Gerner P, et al Effects of early and late diabetic neuropathy on sciatic nerve block duration and neurotoxicity in Zucker diabetic fatty rats. Br J Anaesth. 2015;114:319-26.

7. Ten Hoope W, Looije M, Lirk P. Regional anesthesia in diabetic peripheral neuropathy. Curr Opin Anaesthesiol. 2017;30:627-31.

8. Epstein-Barash H, Shichor I, Kwon AH, Hall S, Lawlor MW, Langer R, et al. Prolonged duration local anesthesia with minimal toxicity. Proc Natl Acad Sci. 2009;106:7125-30.

9. Richard BM, Rickert DE, Newton PE, Ott LR, Haan D, Brubaker AN, et al. Safety Evaluation of EXPAREL (DepoFoam Bupivacaine) Administered by Repeated Subcutaneous Injection in Rabbits and Dogs: Species Comparison. J Drug Deliv. 2011;2011:1-14.

10. Balocco AL, Van Zundert PGE, Gan SS, Gan TJ, Hadzic A. Extended release bupivacaine formulations for postoperative analgesia. Curr Opin Anaesthesiol. 2018;31:636-42.

11. Dyhre H, Söderberg L, Björkman S, Carlsson C. Local Anesthetics in LipidDepot Formulations-Neurotoxicity in Relation to Duration of Effect in a Rat Model. Reg Anesth Pain Med. 2006;31:401-8.

12. Richard BM, Newton P, Ott LR, Haan D, Brubaker AN, Cole PI, et al. The Safety of EXPAREL ${ }^{\otimes}$ (Bupivacaine Liposome Injectable Suspension) Administered by Peripheral Nerve Block in Rabbits and Dogs. J Drug Deliv. 2012;2012:1-10.

13. McAlvin JB, Padera RF, Shankarappa SA, Reznor G, Kwon AH, Chiang HH, et al. Multivesicular liposomal bupivacaine at the sciatic nerve. Biomaterials. 2014:35:4557-64

14. Damjanovska M, Cvetko E, Hadzic A, Seliskar A, Plavec T, Mis K, et al. Neurotoxicity of perineural vs intraneural-extrafascicular injection of liposomal bupivacaine in the porcine model of sciatic nerve block. Anaesthesia. 2015:70:1418-26.

15. Iffeld BM, Viscusi ER, Hadzic A, Minkowitz HS, Morren MD, Lookabaugh J, et al. Safety and Side Effect Profile of Liposome Bupivacaine (Exparel) in Peripheral Nerve Blocks. Reg Anesth Pain Med. 2015;40:572-82.

16. Rwei AY, Sherburne RT, Zurakowski D, Wang B, Kohane DS. Prolonged Duration Local Anesthesia Using Liposomal Bupivacaine Combined With Liposomal Dexamethasone and Dexmedetomidine. Anesth Analg. 2017;126:1170-5.

17. Vandepitte C, Kuroda M, Witvrouw R, Anne L, Bellemans J, Corten K, et al. Addition of liposome bupivacaine to bupivacaine $\mathrm{HCl}$ versus bupivacaine $\mathrm{HCl}$ alone for interscalene brachial plexus block in patients having major shoulder surgery. Reg Anesth Pain Med. 2017;42:334-41.

18. Grazava IG, Rogovaya OS, Borisov MA, Vorotelyak EA, Vasiliev AV. Pathogenesis of type 1 diabetes mellitus and rodent experimental models. Acta Naturae. 2018:10:24-33.

19. Lirk P, Flatz M, Haller I, Hausott B, Blumenthal S, Stevens MF, et al. In Zucker diabetic fatty rats, subclinical diabetic neuropathy increases in vivo lidocaine block duration but not in vitro neurotoxicity. Reg Anesth Pain Med. 2012;37:601-6.

20. Lu J, Xu SY, Zhang QG, Xu R, Lei HY. Bupivacaine induces apoptosis via mitochondria and p38 MAPK dependent pathways. Eur J Pharmacol. 2011; 657:51-8.

21. Yang S, Abrahams MS, Hurn PD, Grafe MR, Kirsch JR. Local Anesthetic Schwann Cell Toxicity Is Time and Concentration Dependent. Reg Anesth Pain Med. 2011;36:444-51.

22. Verlinde $M$, Hollmann $M$, Stevens $M$, Hermanns $H$, Werdehausen $R$, Lirk $P$. Local Anesthetic-Induced Neurotoxicity. Int J Mol Sci. 2016;17:339.

23. Hadzic A, Minkowitz HS, Melson TI, Berkowitz R, Uskova A, Ringold F, et al. Liposome Bupivacaine Femoral Nerve Block for Postsurgical Analgesia after Total Knee Arthroplasty. Anesthesiology. 2016;124:1372-83.

24. Hu D, Onel E, Singla N, Kramer WG, Hadzic A. Pharmacokinetic Profile of Liposome Bupivacaine Injection Following a Single Administration at the Surgical Site. Clin Drug Investig. 2012;33:109-15.

25. Walker D, Carrington A, Cannan SA, Sawicki D, Sredy J, Boulton AJM, et al. Structural abnormalities do not explain the early functional abnormalities in the peripheral nerves of the streptozotocin diabetic rat. J Anat. 1999;195:419-27.

26. Sharma AK, Thomas PK. Peripheral nerve structure and function in experimental diabetes. J Neurol Sci. 1974;23:1-15.
27. Pan H, Ding Y, Yan N, Nie Y, Li M, Tong L. Trehalose prevents sciatic nerve damage to and apoptosis of Schwann cells of streptozotocin-induced diabetic C57BL/6J mice. Biomed Pharmacother. 2018;105:907-14.

28. Murakami T, Iwanaga T, Ogawa Y, Fujita Y, Sato E, Yoshitomi H, et al. Development of sensory neuropathy in streptozotocin-induced diabetic mice. Brain Behav. 2013;3:35-41.

29. Feldman EL, Nave KA, Jensen TS, Bennett DLH. New Horizons in Diabetic Neuropathy: Mechanisms, Bioenergetics, and Pain. Neuron. 2017;93:1296-313.

30. Hidmark AS, Nawroth PP, Fleming T. STZ causes depletion of immune cells in sciatic nerve and dorsal root ganglion in experimental diabetes. J Neuroimmunol. 2017:306:76-82.

31. King AJF. The use of animal models in diabetes research. $\mathrm{Br} J$ Pharmacol 2012;166:877-94.

32. Williams BA. Toward a potential paradigm shift for the clinical care of diabetic patients requiring perineural analgesia: Strategies for using the diabetic rodent model. Reg Anesth Pain Med. 2010;35:329-32.

33. Vital $P$, Larrieta $E$, Hiriart M. Sexual dimorphism in insulin sensitivity and susceptibility to develop diabetes in rats. J Endocrinol. 2006;190:425-32.

34. National Research Council (U.S.). Committee for the Update of the Guide for the Care and Use of Laboratory Animals., Institute for Laboratory Animal Research (U.S.). Guide for the Care and Use of Laboratory Animals. National Academies Press; 2011.

35. Wu KK, Huan Y. Streptozotocin-induced diabetic models in mice and rats. Curr Protoc Pharmacol. 2008:Chap. 5:Unit 5.47.

36. O'Brien PD, Sakowski SA, Feldman EL. Mouse models of diabetic neuropathy. ILAR J. 2014:54:259-72.

37. Allen JW, Yaksh TL. Assessment of acute thermal nociception in laboratory animals. Methods Mol Med. 2004;99:11-23.

38. An K, Elkassabany NM, Liu J. Dexamethasone as adjuvant to bupivacaine prolongs the duration of thermal antinociception and prevents bupivacaine-induced rebound hyperalgesia via regional mechanism in a mouse sciatic nerve block model. PLoS One. 2015;10:e0123459.

39. Hargreaves $K$, Dubner R, Brown F, Flores C, Joris J. A new and sensitive method for measuring thermal nociception in cutaneous hyperalgesia. Pain. 1988:32:77-88.

40. Hole K, Tjølsen A. The tail-flick and formalin tests in rodents: changes in skin temperature as a confounding factor. Pain. 1993:53:247-54.

41. Deuis JR, Dvorakova LS, Vetter I. Methods Used to Evaluate Pain Behaviors in Rodents. Front Mol Neurosci. 2017:10:284.

42. Thalhammer JG, Vladimirova M, Bershadsky B, Strichartz GR. Neurologic evaluation of the rat during sciatic nerve block with lidocaine. Anesthesiology. 1995:82:1013-25.

43. Kohane DS, Lipp M, Kinney RC, Lotan N, Langer R. Sciatic nerve blockade with lipid-protein-sugar particles containing bupivacaine. Pharm Res. 2000;17:1243-9.

44. Mueller M, Wacker K, Ringelstein EB, Hickey WF, Imai Y, Kiefer R. Rapid response of identified resident endoneurial macrophages to nerve injury. Am J Pathol. 2001;159:2187-97.

45. Jolivalt CG, Frizzi KE, Guernsey L, Marquez A, Ochoa J, Rodriguez M, et al. Peripheral Neuropathy in Mouse Models of Diabetes. Curr Protoc Mouse Biol. 2016:6:223-55.

46. Whitlock EL, Brenner MJ, Fox IK, Moradzadeh A, Hunter DA, Mackinnon SE. Ropivacaine-induced peripheral nerve injection injury in the rodent model. Anesth Analg. 2010;111:214-20.

\section{Publisher's Note}

Springer Nature remains neutral with regard to jurisdictional claims in published maps and institutional affiliations.

Ready to submit your research? Choose BMC and benefit from:

- fast, convenient online submission

- thorough peer review by experienced researchers in your field

- rapid publication on acceptance

- support for research data, including large and complex data types

- gold Open Access which fosters wider collaboration and increased citations

- maximum visibility for your research: over $100 \mathrm{M}$ website views per year

At $\mathrm{BMC}$, research is always in progress.

Learn more biomedcentral.com/submissions 